\title{
Viscosity of liquid Ag-In-Sb-Te: Evidence of a fragile-to-strong crossover
}

\author{
J. Orava, ${ }^{1,2, a)}$ H. Weber, ${ }^{3}$ I. Kaban, ${ }^{3}$ and A. L. Greer ${ }^{1,2, a)}$ \\ ${ }^{1}$ Department of Materials Science \& Metallurgy, University of Cambridge, Cambridge CB3 OFS, United Kingdom \\ ${ }^{2}$ WPI Advanced Institute for Materials Research, Tohoku University, Sendai 980-8577, Japan \\ ${ }^{3}$ IFW Dresden, Institute for Complex Materials, Dresden 011 71, Germany
}

The temperature-dependent viscosity $\eta(T)$ is measured for the equilibrium liquid of the chalcogenide Ag-In-Sb-Te (AIST), the first time this has been reported for a material of actual interest for phase-change memory. The measurements, in the range 829-1254 K, are made using an oscillating-crucible viscometer, and show a liquid with high fragility and low viscosity, similar to liquid pure metals. Combining the high-temperature viscosity measurements with values inferred from crystal growth rates in the supercooled liquid allows the form of $\eta(T)$ to be estimated over the entire temperature range from above the melting point down to the glass transition. It is then clear that $\eta(T)$ for liquid AIST cannot be described with a single fragility value, unlike other phase-change chalcogenides such as liquid Ge-Sb-Te. There is clear evidence for a fragileto-strong crossover on cooling liquid AIST, similar to that analyzed in $\mathrm{Te}_{85} \mathrm{Ge}_{15}$. The change in fragility associated with the crossover in both these cases is rather weak, giving a broad temperature range over which $\eta(T)$ is near-Arrhenius. We discuss how such behavior may be beneficial for the performance of phase-change memory. Consideration of the fragile-to-strong crossover in liquid chalcogenides may be important in tuning compositions to optimize device performance.

\section{INTRODUCTION}

In chalcogenide-based phase-change memory (PCM), Joule heating is used to induce reversible transitions: from glass to crystal using weaker electrical pulses; and from crystal to glass, via melting and quenching, using stronger pulses. The same transitions have been exploited for some years in optical discs, such as DVD-RW and Blu-ray ${ }^{\mathrm{TM}}$, where the heating is by laser pulses. ${ }^{1}$ The many studies of optical media suggest that phase-change (PC) chalcogenides fall in two categories: nucleation-dominated, for which the archetype is $\mathrm{Ge}_{2} \mathrm{Sb}_{2} \mathrm{Te}_{5}$ (GST), and growth-dominated, for which the archetype is (Ag,In)-doped $\mathrm{Sb}_{2} \mathrm{Te}$ (AIST). The distinction is based on the crystallization mode of the glassy written marks and is relevant for the speed of data recording. ${ }^{2}$ In nucleation-dominated media, the marks crystallize from many internal nuclei, and in growth-dominated media they do so by growth inwards from the crystalline periphery. Erasure (crystallization) is faster by the first mode for larger marks and by the second mode for smaller marks. ${ }^{3}$

\footnotetext{
a) Authors to whom correspondence should be addressed. Electronic mail: jo316@ cam.ac.uk (JO); alg13@ cam.ac.uk (ALG)
} 
Research on resistive-memory technologies is currently very active. ${ }^{4}$ Among these, PCM is a promising candidate for universal memory. ${ }^{5,6}$ Two conflicting requirements must be met: in memory operation, crystallization times must be short, $\sim 1$ ns, and preferably shorter; ${ }^{5}$ but the memory must also be non-volatile, resistant to spontaneous crystallization, with data retention for $>10$ years at room temperature. Thus the temperature-dependence of crystallization rates is critical; it is now usual to consider this in terms of the fragility $m$ of the supercooled liquid. ${ }^{7}$ The temperature-dependent crystal growth rate $U(T)$ is normally taken to be inversely proportional to the liquid viscosity $\eta(T)$, and the temperature dependence of $\eta$, just above the glass-transition temperature $T_{\mathrm{g}}$, is given by $m=\left[\mathrm{d}\left(\log _{10} \eta\right) / \mathrm{d}\left(T_{\mathrm{g}} / T\right)\right]_{T=T_{\mathrm{g}}}$.

For PC chalcogenides, this description was first demonstrated for GST, where the crystallization kinetics (obtained from ultrafast differential scanning calorimetry, DSC) implied a high fragility, $m \approx 90 .^{8}$ In that work, the form of $\eta(T)$ was fitted using the free-volume model of Cohen and Grest. ${ }^{9}$ The validity of that approach has been questioned; for example, for growth-dominated $\mathrm{Sb}_{93} \mathrm{Ge}_{7}$ (compositions here, and throughout, are in at.\%), the Cohen-Grest model overestimates the measured crystal growth rates by $2-3$ orders of magnitude at intermediate temperatures. ${ }^{10}$ The form of $\eta(T)$ suggested by Mauro et al. ${ }^{11}$ may provide a better description.

The fitting of crystallization kinetics is complicated by the issue, on cooling towards $T_{\mathrm{g}}$, of possible progressive decoupling of crystal growth, and in particular the kinetics-limited (thermodynamically unconstrained) growth rate $U_{\text {kin }}$, from viscous flow; this is characterized by the exponent $\xi$, where $U_{\text {kin }} \propto \eta^{-\xi} .{ }^{12}$ More fragile liquids show greater decoupling (i.e. $\xi$ deviating further below 1). For GST, $\xi=0.67$, and the consequence is that, just above $T_{\mathrm{g}}$, crystal growth is up to $10^{5} \times$ faster than would be predicted from a simple inverse proportionality to $\eta(T) ;^{8}$ this is obviously bad for PCM data retention. The coupling between $\eta$ and $U$ can be strengthened (i.e. $U$ at a given temperature can be decreased) by sandwiching the GST between dielectric layers; ${ }^{13}$ significant stresses develop because of the crystallization shrinkage occurring under constraint. Even so, $U$ remains somewhat decoupled $(\xi=0.80)$ from $\eta .^{13}$

Recently, Orava et al. ${ }^{14}$ showed, from an ultrafast DSC study of crystallization, that the form of $\eta(T)$ for supercooledliquid AIST is quite different from that for GST. It has an extended region of Arrhenius temperature dependence, first found in optical measurements of crystallization rate by Salinga et al. ${ }^{15}$ who noted that decoupling does not help in fitting the observed behavior. Orava et al. ${ }^{14}$ suggested that the form of $\eta(T)$ could be explained only by invoking a fragile-to-strong crossover on cooling the liquid. They furthermore suggested that such a crossover could explain the distinction between nucleation- and growth-dominated media, and that it would be relevant for the operation of PCM: the fragile (low $\eta$, high 
mobility) behavior at high temperature is helpful for fast switching, while the strong behavior at low temperature helps nonvolatility by retarding crystallization, not only through higher $\eta$, but also through reduced or negligible decoupling of crystal growth from viscous flow.

The current study presents the first direct measurements of $\eta(T)$ in an actual PCM material. We examine the fragile-tostrong crossover in AIST $\left(\mathrm{Ag}_{5.5} \mathrm{In}_{6.5} \mathrm{Sb}_{59} \mathrm{Te}_{29}\right)$, and aim to better characterize this phenomenon that could be so important for PCM operation.

\section{RESULTS}

Figure 1 shows $\eta(T)$ measured from $1254 \mathrm{~K}$ down to solidification at different cooling rates $(\Phi)$ which are limited by the experimental set-up (See Materials and Methods, Sec. V). The $\eta(T)$ values are independent of $\Phi$, confirming that they represent an equilibrium liquid. The sharp decrease in apparent viscosity at lower temperatures is because partial or full crystallization reduces the viscous-flow-related damping in the sample. The onset of crystallization is consistent with a liquidus temperature of $817 \mathrm{~K}$, as determined by Kalb et al. ${ }^{16}$ AIST is not a good glass-former: the critical cooling rate for glass formation may be as high as $10^{9}-10^{11} \mathrm{~K} \mathrm{~s}^{-1}$. It is then unsurprising that, at the cooling rates $83-45 \mathrm{mK} \mathrm{s}$ in the present work, heterogeneous nucleation of crystallization on the container walls could not be avoided and that no significant supercooling of the liquid could be achieved. (Supercoolings up to $\Delta T \approx 60 \mathrm{~K}$ can be achieved under a $\mathrm{B}_{2} \mathrm{O}_{3}$ flux, ${ }^{17}$ but the use of a flux would invalidate the viscometry in the present work).

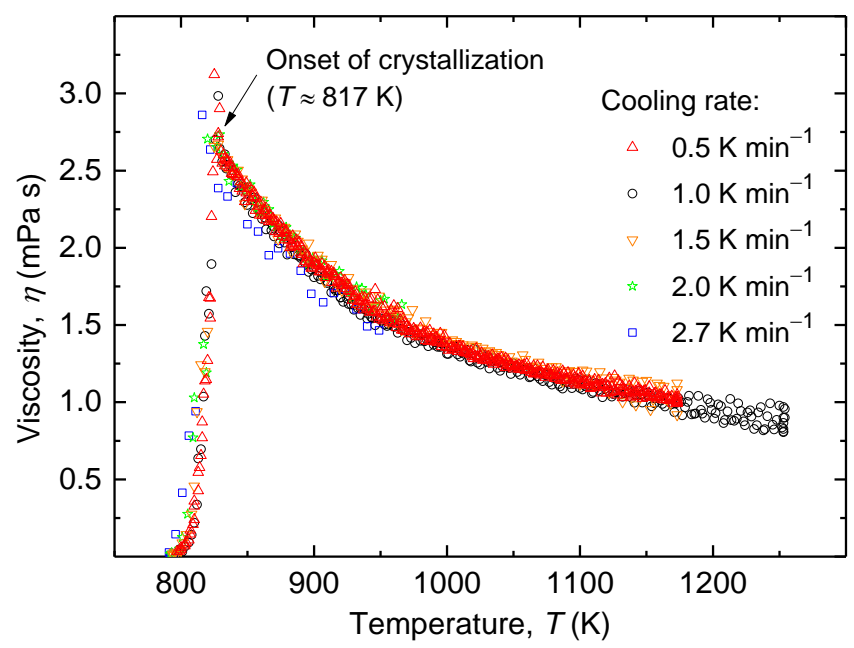

FIG. 1. Temperature-dependent viscosity $\eta(T)$ of liquid $\mathrm{Ag}_{5.5} \mathrm{In}_{6.5} \mathrm{Sb}_{59} \mathrm{Te}_{29}$ measured at the cooling rates shown. 
In the following analyses, we use the viscosity values measured at $\Phi=1 \mathrm{~K} \mathrm{~min}^{-1}$ (Figure 1, values are listed in Table 1).

The measured viscosity of $\log _{10} \eta(\mathrm{Pa} \mathrm{s})=-2.64$ at $T=850 \mathrm{~K}$ (Table I) is close to the value $\log _{10} \eta(\mathrm{Pa} \mathrm{s})=-3.11$ obtained by molecular-dynamics simulations of liquid $\mathrm{Ag}_{3.5} \mathrm{In}_{3.8} \mathrm{Sb}_{75.0} \mathrm{Te}_{17.7}{ }^{18}$ The $\eta(T)$ of AIST closely resembles measurements ${ }^{19}$ on $\mathrm{Te}_{85} \mathrm{Ge}_{15}$, both liquids having a high-temperature viscosity similar to that for pure liquid metals. ${ }^{20}$

Table I. Average measured values of $\eta(T)$ in $\mathrm{mPa}$ s at the cooling rate of $1 \mathrm{~K} \mathrm{~min}^{-1}$ (Fig. 1). The error in measured viscosity is less than $10 \%$.

\begin{tabular}{|c|c|c|c|c|c|c|c|c|c|c|c|c|c|}
\hline $\begin{array}{c}T \\
(\mathrm{~K})\end{array}$ & $\begin{array}{c}\eta \\
(\mathrm{mPas})\end{array}$ & $\begin{array}{c}T \\
(\mathrm{~K})\end{array}$ & $\begin{array}{c}\eta \\
(\mathrm{mPas})\end{array}$ & $\begin{array}{c}T \\
(\mathrm{~K})\end{array}$ & $\begin{array}{c}\eta \\
(\mathrm{mPas})\end{array}$ & $\begin{array}{c}T \\
(\mathrm{~K})\end{array}$ & $\begin{array}{c}\eta \\
(\mathrm{mPas})\end{array}$ & $\begin{array}{c}T \\
(\mathrm{~K})\end{array}$ & $\begin{array}{c}\eta \\
(\mathrm{mPas})\end{array}$ & $\begin{array}{c}T \\
(\mathrm{~K})\end{array}$ & $\begin{array}{c}\eta \\
(\mathrm{mPas})\end{array}$ & $\begin{array}{c}T \\
(\mathrm{~K}) \\
\end{array}$ & $\begin{array}{c}\eta \\
(\mathrm{mPas})\end{array}$ \\
\hline 829 & 2.644 & 890 & 1940 & 961 & 1480 & 1022 & 1301 & 1082 & 1171 & 1142 & 1.047 & 1202 & \\
\hline 831 & 2.559 & 893 & 005 & 963 & & 1023 & & & & & & 1204 & \\
\hline 833 & 606 & 4 & & 965 & & 1 & & & & 46 & & 206 & \\
\hline 834 & 2.513 & 896 & & 966 & & 1027 & & & & 147 & & 208 & \\
\hline 836 & 2.539 & 898 & & 968 & & 1028 & & & & 1149 & & 1209 & \\
\hline 838 & 2.473 & 900 & & 970 & 1401 & 1030 & 1.260 & 1090 & & 151 & 62 & 1211 & 41 \\
\hline 839 & 2.502 & 903 & & 971 & & 1032 & & 1092 & & 1152 & & 1213 & 21 \\
\hline 841 & 2.360 & 905 & & 973 & & 1033 & & 1094 & & 54 & & 214 & \\
\hline 843 & 2.413 & 908 & & 975 & 1.4 & 1035 & & 1095 & & 1156 & & 1216 & 79 \\
\hline 844 & 2.400 & 910 & & 976 & & & & & & & & & \\
\hline 846 & 2.394 & 913 & & 978 & & 1038 & & 1099 & & 59 & & 1219 & 93 \\
\hline 848 & 2.295 & 915 & & 980 & & & & & & & & 1221 & \\
\hline 849 & & 916 & & 981 & & & & & & & & & \\
\hline 850 & 2.278 & 918 & 1.724 & 983 & 1 & 1043 & 51 & 1104 & & 1164 & & 1224 & 50 \\
\hline 853 & & 920 & & 985 & & & & & & & & 26 & \\
\hline 854 & 2.225 & 922 & & 986 & 1.3 & 1047 & & 1107 & & 1167 & & 1228 & 48 \\
\hline 855 & 2.325 & 925 & & 988 & & & & & & & & 229 & \\
\hline 858 & 2.241 & 926 & 1. & 990 & & 1050 & & 1110 & & 1171 & & 1231 & 26 \\
\hline 859 & 2.243 & 928 & & 991 & & 10 & & & & 72 & & 1233 & \\
\hline 861 & 2.186 & 930 & & & & & & & & & & 4 & \\
\hline 863 & 2.195 & 933 & & 995 & & 10 & & 11 & & 1176 & & 1236 & \\
\hline 864 & & 935 & & & & & & & & & & & \\
\hline 866 & 2.210 & 938 & & 998 & & & & 1119 & & 1179 & & 1239 & 25 \\
\hline 868 & 2.113 & 940 & 1.5 & 1000 & 1.3 & 1060 & 21 & 1120 & & 81 & & 1241 & 09 \\
\hline 869 & 2.140 & 941 & & 1001 & & 10 & & 1122 & & & & 1243 & \\
\hline 870 & 2.074 & 943 & & 1003 & & 1063 & & 1124 & & 84 & & 1245 & 54 \\
\hline 873 & 2.149 & 944 & & & & & & & & & & 1246 & \\
\hline 874 & 2.029 & 946 & & 1007 & & & & 1127 & & & & 1248 & \\
\hline 875 & & 948 & & & & & & & & & & & \\
\hline 878 & 2.033 & 950 & & & & & & 1131 & & 1191 & & 1252 & 0.836 \\
\hline 879 & 2.064 & 951 & 1. & 1011 & 1.3 & 1072 & 37 & 11 & 1. & 1192 & 26 & 1253 & 0.863 \\
\hline 880 & 1.955 & 953 & & & & & & 1134 & & & & 1254 & 0.888 \\
\hline 883 & 2.051 & 955 & & 1015 & 1.339 & 1075 & & & & 1196 & 1.019 & & \\
\hline 884 & & 956 & & & & & & & & & & & \\
\hline 885 & & 958 & & 1018 & & & & 1139 & & 1199 & & & \\
\hline 888 & 1.949 & 960 & 1.489 & 1020 & 1.344 & 1080 & 1.157 & 1141 & 0.997 & 1201 & 0.894 & & \\
\hline
\end{tabular}

As already noted, liquids characterized by a single fragility value, i.e. not showing a fragile-to-strong crossover, can be described, as proposed by Mauro et al., ${ }^{11}$ using an equation based on Adam-Gibbs theory. ${ }^{21}$ This MYEGA equation is: 


$$
\log _{10} \eta(T)=\log _{10} \eta_{\infty}+\left(\frac{B}{T}\right) \exp \left(\frac{C}{T}\right)
$$

where $\eta_{\infty}$ is the high-temperature limit of viscosity, and $B$ and $C$ are adjustable parameters. Equation 1 can fit $\eta(T)$ for a variety of supercooled liquids with fragilities $m$ ranging from 20 to $90 .^{11}$ The fits are much better than is possible with the classical Vogel-Fulcher-Tammann expression. ${ }^{11}$

We first fit the viscosity data (Table 1) only. In Equation 1, the fitted parameters are: $\log _{10} \eta_{\infty}(\mathrm{Pa} s)=-3.31 \pm 0.02, B=$ $100 \pm 16 \mathrm{~K}$ and $C=1487 \pm 112 \mathrm{~K}$, with quality of fit $R^{2}=0.9872$. This fit to Equation 1 , when extrapolated, comes close to the accepted glass transition at $378 \mathrm{~K}$ : the conventional value of $\log _{10} \eta(\mathrm{Pa} \mathrm{s})=12$ is reached at $368 \mathrm{~K}$, or equivalently the viscosity at $378 \mathrm{~K}$ is underestimated by (just) two orders of magnitude. The corresponding high-temperature fragility, $m^{\prime}$ in the terminology adopted by Zhang et al., ${ }^{22}$ is $\sim 74$.

While viscosity data are not available for the supercooled liquid, values can be inferred from crystal growth rates, and we use data from as-deposited single-film $\mathrm{Ag}_{5.5} \operatorname{In}_{6.5} \mathrm{Sb}_{59} \mathrm{Te}_{29}$ (Fig. 2). Values of $U_{\text {kin }}(T)$ obtained from ultrafast calorimetry and numerical modeling ${ }^{14}$ can be transposed on to the Angell plot. Atomic force microscopy observations of crystal size give growth rates, ${ }^{23}$ from which $\eta(T)$ can be inferred via the Stokes-Einstein relation. Two approaches were adopted to fit the combined data. In the first, $T_{\mathrm{g}}$ is taken to be $378 \mathrm{~K}^{14}$ and the fit (Fig. 2, black dotted line) adopts the conventional value, $\log _{10} \eta(\mathrm{Pa} \mathrm{s})=12$, at that point; the fitted parameters are: $\log _{10} \eta_{\infty}(\mathrm{Pa} \mathrm{s})=-4.31 \pm 0.06, B=617 \pm 36 \mathrm{~K}$ and $C=890 \pm 23 \mathrm{~K}$ with quality of fit $R^{2}=0.9919$. The second approach, commonly adopted in the literature, treats $T_{\mathrm{g}}$ as an adjustable parameter and yields an estimate, in this case, of $400 \mathrm{~K}$; the fitted values are: $\log _{10} \eta_{\infty}(\mathrm{Pa} \mathrm{s})=-3.64 \pm 0.03, B=198 \pm 11 \mathrm{~K}$ and $C=$ $1382 \pm 24 \mathrm{~K}$, with $R^{2}=0.9980$ (Fig. 2, grey dashed line). The value of $T_{\mathrm{g}}$ appears high (400 K compared to $\left.378 \mathrm{~K}\right)$ and the fragility near $T_{\mathrm{g}}$ is overestimated (the grey dashed line in Fig. 2 corresponds to $m \approx 70$ ). Similar fitting problems near $T_{\mathrm{g}}$ may account for other anomalously high fragility values reported for liquid AIST: $m \approx 130$ for melt-quenched films, ${ }^{15}$ and $m \approx 104$ \& 217 for as-deposited and melt-quenched films. ${ }^{24}$

Each of these single-fragility fits (using Equation 1) matches the slope of the indirectly determined $\eta(T)$ values in the range $T_{\mathrm{g}} / T=0.77-0.91$ in the Angell plot. To test whether these approaches can provide a good fit to the data over the full range in Fig. 2, i.e. $T_{\mathrm{g}} / T=0.3-1$, we examine the high-temperature range more closely (Fig. 3 ); the new $\eta(T)$ data, directly measured in the present work, are then particularly relevant. When $T_{\mathrm{g}}$ is set at $378 \mathrm{~K}$, the fitted curve (Fig. 3 , black dotted 
line) has a slope much higher, by factor of $\sim 2.5$, than that of the measured data themselves. When $T_{\mathrm{g}}$ is treated as an adjustable parameter, the fitted curve (Fig. 3, grey dashed line) is closer to the measured data, but its slope is still $\sim 1.7$ times greater than that of the data (and, as noted above, the fit to the lower-temperature data is worsened). Figure 3 shows that although the fitted and measured values of $\eta(T)$ match at $\left(T_{\mathrm{g}} / T\right)=0.364$, and never deviate by more than a factor of $\sim 2.2$, the slopes (temperature dependences) are irreconcilably different.

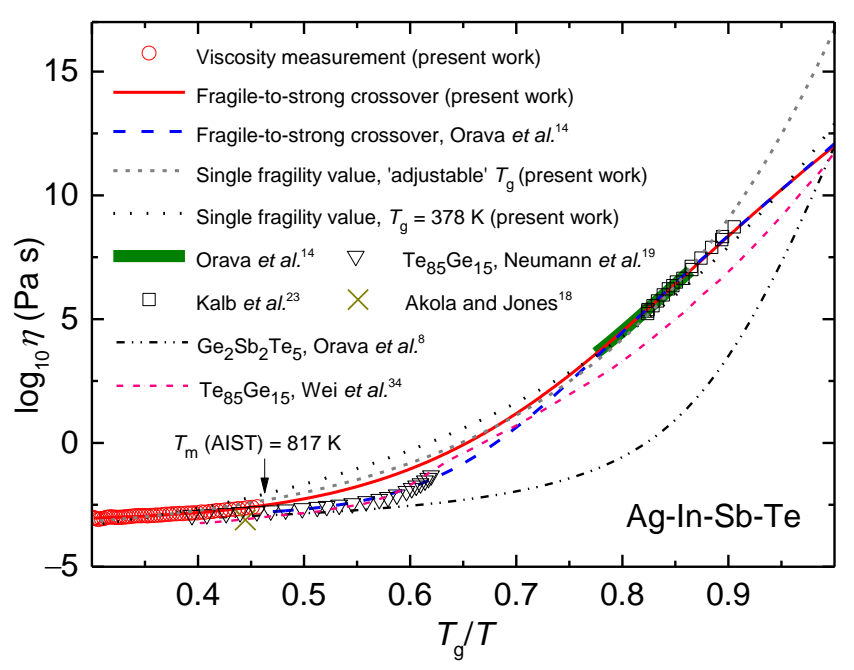

FIG. 2. Angell plot for the temperature-dependent viscosity of AIST and related systems. Measurements of viscosity of equilibrium liquid AIST cooled at $\Phi=1 \mathrm{~K} \mathrm{~min}^{-1}$ (Fig. 1) are shown by open red circles (some data points omitted for clarity). The intermediate-temperature viscosities of AIST are calculated from crystal growth velocities in as-deposited single-film $A_{{ }_{5.5}} \mathrm{In}_{6.5} \mathrm{Sb}_{59} \mathrm{Te}_{29}$, measured by Kalb et al. ${ }^{23}$ (open black squares) and by Orava et al. ${ }^{14}$ (olive line). The black and grey dashed lines show the best single-fragility fits, using Equation 1 , by (i) setting $T_{\mathrm{g}}=378 \mathrm{~K}, \log _{10} \eta(\mathrm{Pa} \mathrm{s})=12$, or (ii) by taking $T_{\mathrm{g}}$ as an adjustable parameter. The dashed blue line shows the best fit to the generalized-MYEGA model (Equation 2) obtained without the present viscosity data. ${ }^{14}$ The solid red line shows the equivalent best fit when the viscosity data are included. These fits incorporate a fragile-to-strong crossover. The viscosity (dark yellow cross) of $\mathrm{Ag}_{3.5} \mathrm{In}_{3.8} \mathrm{Sb}_{75.0} \mathrm{Te}_{17.7}$ at $850 \mathrm{~K}$ is from molecular-dynamics simulations. ${ }^{18}$ For $\mathrm{Te}_{85} \mathrm{Ge}_{15}$, viscosity data ${ }^{19}$ (open triangles), when combined with fitting to calorimetric data, also show a fragile-to-strong crossover (pink dot-dashed line). ${ }^{34}$ The black dash-dot-dot line shows $\eta(T)$ of supercooled liquid calculated from growth velocities in as-deposited single-film $\mathrm{Ge}_{2} \mathrm{Sb}_{2} \mathrm{Te}_{5}{ }^{8}{ }^{8}$

The fragile-to-strong crossover in supercooled metallic-glass-forming liquids has been described using a modification of Equation $1 .^{22}$ This generalized-MYEGA equation is:

$$
\log _{10} \eta(T)=\log _{10} \eta_{\infty}+\frac{1}{T\left[W_{1} \exp \left(-\frac{C_{1}}{T}\right)+W_{2} \exp \left(-\frac{C_{2}}{T}\right)\right]}
$$


where $\eta_{\infty}$ has the same meaning as in Equation 1, and $W_{1}, C_{1}, W_{2}$ and $C_{2}$ are fitting parameters. Orava et al. ${ }^{14}$ used Equation 2 to fit their suggested fragile-to-strong crossover in liquid AIST. In the absence of high-temperature measurements, they proposed an approximate description of $\eta(T)$ for AIST (Fig. 2, dashed blue line), suggesting a broad fragile-to-strong crossover; the fitted values in Equation 2 are: $\log \eta_{\infty}(\mathrm{Pa} \mathrm{s})=-2.95 \pm 0.04, W_{1}=5.3 \pm 2.2 \mathrm{~K}^{-1}, C_{1}=5334 \pm 231 \mathrm{~K}, W_{2}=$ $(5.79 \pm 0.71) \times 10^{-4} \mathrm{~K}^{-1}$ and $C_{2}=459 \pm 45 \mathrm{~K}$, with $R^{2}=0.9997$.

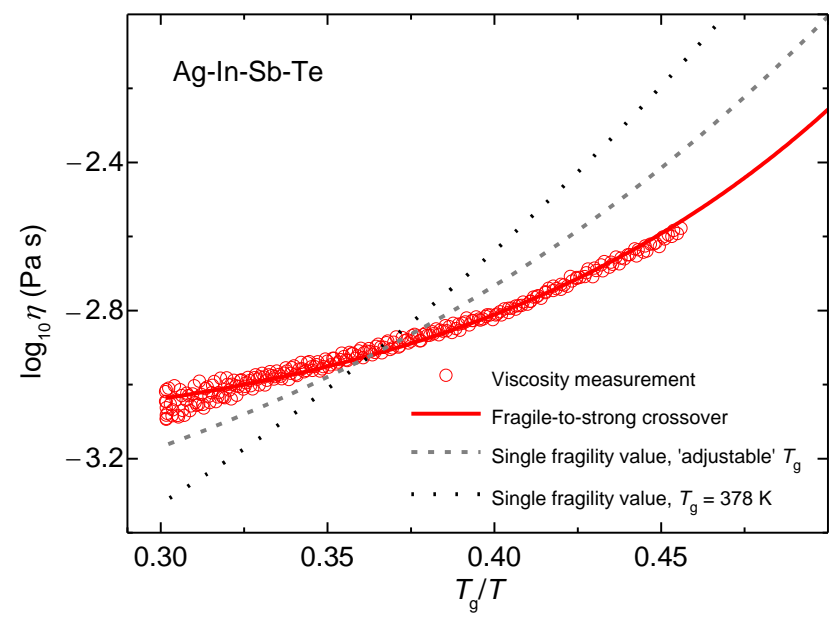

FIG. 3. Close-up view of the high-temperature data and best-fit viscosity models from Figure 2. The open red circles show the measured data for the viscosity of equilibrium liquid AIST cooled at $\Phi=1 \mathrm{~K} \mathrm{~min}^{-1}$ (Table 1). The black dotted and grey dashed lines show, respectively, the best single-fragility fits, using Equation 1, (i) by setting $T_{\mathrm{g}}=378 \mathrm{~K}, \log _{10} \eta(\mathrm{Pa} \mathrm{s})=12$, or (ii) by taking $T_{\mathrm{g}}$ as an adjustable parameter. The solid red line shows the best fit to the generalized-MYEGA model (Equation 2), incorporating a fragile-to-strong crossover.

This approach can be improved by including the high-temperature $\eta(T)$ from the oscillating-crucible measurements in the present work. The best-fit values then are: $\log _{10} \eta_{\infty}(\mathrm{Pa} \mathrm{s})=-3.16 \pm 0.01, W_{1}=(6.4 \pm 0.3) \times 10^{-2} \mathrm{~K}^{-1}, C_{1}=2949 \pm 34 \mathrm{~K}, W_{2}=$ $(1.59 \pm 0.14) \times 10^{-4} \mathrm{~K}^{-1}$ and $C_{2}=27 \pm 29 \mathrm{~K}$, with $R^{2}=0.9999$ (red solid line in Fig. 2 and in close-up, high-temperature, view in Fig. 3). There is a high uncertainty $(100 \%)$ in the $C_{2}$ value, arising from the wide temperature range of Arrhenius kinetics down to $T_{\mathrm{g}}$. Over the range $T_{\mathrm{g}} / T=0.46-0.77$ (i.e. $\left.T=(1.01-0.60) T_{\mathrm{m}}\right)$, in which the Angell plot has its greatest curvature, data are still lacking. Even so, the present viscosity measurements provide direct support for the fragile-to-strong crossover in liquid AIST proposed by Orava et al. ${ }^{14}$

The forms of $\eta(T)$ suggested by Orava et al. ${ }^{14}$ and obtained in the present work (Fig. 2, red solid and blue dashed lines) show a significant difference over $540-756 \mathrm{~K}$, with the largest deviation of $\Delta \log _{10} \eta \approx 0.8$ at $630 \mathrm{~K}$. Both forms show a broad temperature range of Arrhenius kinetics, corresponding to a moderately strong ('intermediate') liquid, with $m \approx 37$, at $T_{\mathrm{g}}$. (We contrast this fragility with the various much higher values quoted in the literature noted above.) 


\section{DISCUSSION}

\section{A. Fragile-to-strong crossover in chalcogenides}

A fragile-to-strong crossover is found in many glass-forming liquids. ${ }^{25}$ In chalcogenides, it may be associated with a polyamorphic transition between a high-temperature metallic and a low-temperature semiconducting liquid, ${ }^{19,26,27}$ and similar transitions can also be induced by pressure and by light. ${ }^{27,28,29}$ The nature of the transition, including its temperature range, may be tuned by varying the composition. ${ }^{26,27,30}$ In chalcogenides for PCM, the resistivity change resulting from such a transition would be directly relevant for device operation.

Another manifestation of changes in structure and bonding in supercooled liquids is hysteresis in viscosity, which can be detected even in the absence of a clear first-order transition. In a particular temperature range, the viscosity values on cooling are lower than those on heating. Such effects have been seen in metallic-glass-forming liquids. ${ }^{31}$ For chalcogenides, early measurements suggested such effects in tellurium, ${ }^{32}$ and recently Sosso et al. ${ }^{33}$ have detected hysteresis in their moleculardynamics simulations of liquid TeGe.

Orava et al. ${ }^{14}$ suggested that $\mathrm{Te}_{85} \mathrm{Ge}_{15}$ shows a fragile-to-strong crossover similar to that in AIST. This suggestion is clearly supported by a recent study, ${ }^{34}$ which analyzed viscosity data ${ }^{19}$ together with newly acquired calorimetric data; AdamGibbs fitting to the combined data gave a consistent form of $\eta(T)$ with a fragile-to-strong crossover (Fig. 2). Interestingly, $\mathrm{Te}_{85} \mathrm{Ge}_{15}$ shows a sharp increase in resistivity as it is cooled. ${ }^{19}$

It is of interest to compare the magnitude of the property change associated with the crossovers seen in different classes of glass-forming liquids. Zhang et al. ${ }^{22}$ quantified this as the ratio of the fragilities on either side of the crossover: $m^{\prime}$ obtained by fitting high-temperature data, and $m$ by fitting just above $T_{\mathrm{g}}$. For $\mathrm{Te}_{85} \mathrm{Ge}_{15},{ }^{34} m^{\prime} \approx 90$ and $m \approx 50$. For AIST, $m=$ $37 ;^{14}$ the present work confirms this value, and fitting of the new high-temperature $\eta(T)$ data gives $m^{\prime}=74$. Thus for AIST, as for $\mathrm{Te}_{85} \mathrm{Ge}_{15},\left(m^{\prime} / m\right) \approx 2$. In water, this ratio is 4.5 , and in metallic-glass-forming liquids it is as high as $8.2{ }^{22,35}$ Hence the crossover in the chalcogenides is comparatively weak, giving a wide temperature range of near-Arrhenius behavior. Near $T_{\mathrm{g}}$, AIST and $\mathrm{Te}_{85} \mathrm{Ge}_{15}$ can be classified as 'intermediate' liquids, and their comparatively low fragility may help to explain why the glass transition can be so difficult to detect calorimetrically in such systems (Ref. ${ }^{8}$, Suppl. Info.). 


\section{B. Effect of fragile-to-strong crossover on crystal growth rates}

The fragility of the supercooled liquid is important in enabling PC chalcogenides to combine (i) fast crystallization during memory switching and (ii) long-term data retention. A fragile-to-strong crossover can affect the relative rates of crystal nucleation and growth and thus be relevant for determining whether a PC chalcogenide is considered nucleation- or growth-dominated. ${ }^{14}$

Here we focus on the possible effect of a crossover on the maximum growth rate $U_{\max }$. Considering a diverse range of glass-forming liquids, $U_{\max }$ is higher for lower reduced glass-transition temperature $\left(T_{\mathrm{rg}}=T_{\mathrm{g}} / T_{\mathrm{m}}\right)$ and for higher $m \cdot{ }^{36}$ The influence of $T_{\mathrm{rg}}$ is the stronger, and there is a good correlation (Fig. $3 \mathrm{~b}$ in Ref. ${ }^{36}$ ) between $U_{\max }$ and the single parameter $T_{\mathrm{gu}}=$ $T_{\mathrm{rg}}-(m / 505)$. This correlation has considered only liquids each characterized by a single value of $m$; we now examine the case of AIST.

In as-deposited thin-film AIST, the maximum in crystal growth rate has been reported as $U_{\max } \approx 1 \mathrm{~m} \mathrm{~s}^{-1}$ at a homologous temperature of $\sim 0.89 T_{\mathrm{m}}$ (from ultrafast calorimetry ${ }^{14}$ ) and as $\sim 2.5 \mathrm{~m} \mathrm{~s}^{-1}$ at $\sim 0.90 T_{\mathrm{m}}$ (from reflectance measurements after femtosecond optical excitation ${ }^{24}$ ). Taking $\eta(T)$ from the present work (red solid line in Fig. 2), and deriving the growth rate from this as in Ref. ${ }^{14}$, gives $U_{\max } \approx 0.1 \mathrm{~m} \mathrm{~s}^{-1}$ at $\sim 0.89 T_{\mathrm{m}}$. The homologous temperature at which the growth rate is maximum $\left(T_{\mathrm{g}} / T=0.52\right.$, Figure 2$)$ is on the high-temperature, fragile side of the crossover, and is significantly higher than would be expected for fast-crystallizing systems characterized by a single fragility. ${ }^{36}$

For AIST, we take $T_{\mathrm{g}}=378 \mathrm{~K}^{14}$ and $T_{\mathrm{m}}=817 \mathrm{~K}^{16}$, giving $T_{\mathrm{rg}}=0.46$. With $m=37, T_{\mathrm{gu}}=0.39$, which from the correlation in Ref. ${ }^{36}$ suggests $U_{\max } \approx 0.3 \mathrm{~m} \mathrm{~s}^{-1}$. With $m=74, T_{\mathrm{gu}}=0.31$, and the correlation suggests $U_{\max } \approx 6 \mathrm{~m} \mathrm{~s}^{-1}$. Thus, within the limited extent of the crossover in this PC chalcogenide, the values of $U_{\max }$ in as-deposited thin films are in broad agreement with the existing correlation. Much higher values of $U_{\max }$, up to $110 \mathrm{~m} \mathrm{~s}^{-1}$, have been noted for melt-quenched samples. ${ }^{24}$ Such values lie outside the correlation, and indeed are higher than would be expected for any system other than pure metals; ${ }^{36}$ further work may be required to interpret crystallization rates in such cases.

All of the quoted values of $U_{\max }$ are high, for example being greater than that for polymorphic growth in the metallicglass-forming system $\mathrm{Zr}_{50} \mathrm{Cu}_{50}$, for which $U_{\max } \approx 2.3 \times 10^{-2} \mathrm{~m} \mathrm{~s}^{-1}$ at $\sim 0.83 T_{\mathrm{m}}{ }^{37}$ 


\section{CONCLUSIONS}

In the operation of phase-change memory (PCM), the temperature-dependent viscosity $\eta(T)$ of the liquid chalcogenide is important in controlling the rate of crystal growth $U$. We provide the first report of direct measurements of $\eta(T)$ for an actual PCM system, namely AIST. The high-temperature, (1.0-1.5) $T_{\mathrm{m}}$, measurements show low $\eta$ values, comparable to those of liquid pure metals, that are independent of cooling rate and represent the equilibrium liquid. Fitting the values to the MYEGA equation suggests a high-temperature fragility $m^{\prime} \approx 74$. Single-fragility models cannot, however, at the same time describe the shape of the present measured temperature dependence of viscosity combined with the crystal growth rates in the range $(0.51-0.60) T_{\mathrm{m}}$; this failure provides direct support for the suggestion that AIST shows a fragile-to-strong crossover on cooling. All the relevant data for $\eta(T)$ in AIST can be fitted with a generalized MYEGA equation, and this gives a lowtemperature (just above $T_{\mathrm{g}}$ ) fragility $m=37$. The ratio $\left(m^{\prime} / m\right) \approx 2$ agrees closely with that recently determined for $\mathrm{Te}_{85} \mathrm{Ge}_{15}$ and supports the suggestion that these two chalcogenide systems have similar forms of $\eta(T)$. This ratio, describing the magnitude of the change associated with the crossover, is among the lowest reported for glass-forming liquids, and results in a broad range over which $\eta(T)$ has a near-Arrhenius temperature dependence. The fragile-to-strong crossover in chalcogenide liquids can be tuned by varying the composition, and this may be important in optimizing PCM performance. The present results suggest that a weak crossover, like that in AIST, may aid long-term data retention near room temperature, while still permitting the fast crystal growth required for fast switching in memory operation.

\section{MATERIALS AND METHODS}

Sample preparation: A 30-g ingot of $\mathrm{Ag}_{5.5} \mathrm{In}_{6.5} \mathrm{Sb}_{59} \mathrm{Te}_{29}$ (at. \%) was synthesized from pure elements of (4N Ag, $5 \mathrm{~N} \mathrm{In,} \mathrm{Sb}$ and $\mathrm{Te}$ ) in a fused-silica ampoule, inner diameter $22.5 \mathrm{~mm}$, which was evacuated, back-filled with an argon inert atmosphere of $250 \mathrm{mbar}$, and sealed. The alloy was melted by heating at $5 \mathrm{~K} \mathrm{~min}^{-1}$ and homogenized at $1254 \mathrm{~K}$ for 3 hours.

Viscosity measurement: The high-temperature viscosity $\eta(T)$ was measured using an oscillating crucible viscometer as described in detail by Gruner and Hoyer. ${ }^{38}$ The internal friction of the liquid causes damping in the induced oscillation, which is monitored by a laser beam photodiode unit. By simulating the measured oscillations using the method of Roscoe and Bainbridge ${ }^{39}$ as modified by Brooks et al., ${ }^{40} \eta(T)$ can be obtained (Fig. 1). The temperature-dependent density $\left(\mathrm{g} \mathrm{cm}^{-3}\right)$ of the liquid, using the ideal-solution approximation and taking densities of the pure elements as in Refs. ${ }^{41,42}$, was calculated to be $\rho(T)=6.817_{(273 \mathrm{~K})}-0.000789 T$. (An alternative expression, $\rho(T)=6.690_{(273 \mathrm{~K})}-0.00057 T, \rho(T)$ was also tested but the 
difference, $\sim 1 \%$, in the calculated $\eta(T)$ was not significant.) The sealed ampoule containing the AIST sample was inserted in

the experimental system which was kept under a vacuum of $10^{-6}-10^{-5}$ mbar. The sample was heated up to $\sim 1254 \mathrm{~K}$, held at this temperature for 1 hour to homogenize, and then $\eta(T)$ was measured at different cooling rates, $\Phi=0.5-2.7 \mathrm{~K} \mathrm{~min}{ }^{-1}$. The temperature was measured, to an accuracy better than $\pm 5 \mathrm{~K}$, by a K-type thermocouple $0.5 \mathrm{~cm}$ under the sample container. For each temperature, by averaging over 10 to 12 oscillations, the error in $\eta(T)$ is less than $10 \%$.

\section{ACKNOWLEDGMENTS}

JO and ALG acknowledge financial support by the World Premier International Research Center Initiative (WPI), MEXT, Japan. All data accompanying this publication are directly available within the publication.

\section{REFERENCES}

${ }^{1}$ V. L. Deringer, R. Dronskowski, and M. Wuttig, Adv. Funct. Mater. 25, 6346 (2015).

${ }^{2}$ B.-S. Lee, R. M. Shelby, S. Raoux, C. T. Retter, G. W. Burr, S. N. Bogle, K. Darmawikarta, S. G. Bishop, and J. R. Abelson, J. Appl. Phys. 115, 063506 (2014).

${ }^{3}$ G.-F. Zhou, Mater. Sci. Eng. A 304-306, 93, (2001).

${ }^{4}$ S. Menzel, U. Böttger, M. Wimmer, and M. Salinga, Adv. Funct. Mater. 25, 6306, (2015).

${ }^{5}$ D. Loke, T. H. Lee, W. J. Wang, L. P. Shi, R. Zhao, Y. C. Yeo, T. C. Chong, and S. R. Elliott, Science 336, 1566 (2012).

${ }^{6}$ S. R. Elliott, Int. J. Appl. Glass Sci. 6, 15 (2015).

${ }^{7}$ C. A. Angell, Science 267, 1924 (1995).

${ }^{8}$ J. Orava, A. L. Greer, B. Gholipour, D. W. Hewak, and C. E. Smith, Nat. Mater. 11, 279 (2012).

${ }^{9}$ M. H. Cohen and G. S. Grest, Phys. Rev. B 20, 1077 (1979).

${ }^{10}$ B. Chen, J. Momand, P. A. Vermeulen, and B. J. Kooi, Cryst. Growth Des. 16, 242 (2016).

${ }^{11}$ J. C. Mauro, Y. Yue, A. J. Ellison, P. K. Gupta, and D. C. Allan, Proc. Nat. Acad. Sci. 106, 19780 (2009).

${ }^{12}$ M. D. Ediger, P. Harrowell, and L. Yu, J. Chem. Phys. 28, 34709 (2008).

${ }^{13}$ J. Orava, A. L. Greer, B. Gholipour, D. W. Hewak, and C. E. Smith, Appl. Phys. Lett. 101, 091906 (2012).

${ }^{14}$ J. Orava, D. W. Hewak, and A. L. Greer, Adv. Funct. Mater. 25, 4851 (2015).

${ }^{15}$ M. Salinga, E. Carria, A. Kaldenbach, M. Bornhöfft, J. Benke, J. Mayer, and M. Wuttig, Nat. Commun. 4, 2371 (2013).

${ }^{16}$ J. Kalb, F. Spaepen, and M. Wuttig, J. Appl. Phys. 93, 2389 (2003).

${ }^{17}$ J. A. Kalb, F. Spaepen, and M. Wuttig, J. Appl. Phys. 98, 054910 (2005).

${ }^{18}$ J. Akola and R. O. Jones, Appl. Phys. Lett. 94, 251905 (2009).

${ }^{19}$ H. Neumann, F. Herwig, and W. Hoyer, J. Non-Cryst. Solids 205-207, 438 (1996).

${ }^{20}$ T. Ishikawa, P.-F. Paradis, J. T. Okada, and Y. Watanabe, Meas. Sci. Technol. 23, 025305 (2012).

${ }^{21}$ G. Adam and J. H. Gibbs, J. Chem. Phys. 43, 139 (1965).

${ }^{22}$ C. Zhang, L. Hu, Y. Yue, and J. C. Mauro, J. Chem. Phys. 133, 014508 (2010).

${ }^{23}$ J. Kalb, F. Spaepen, and M. Wuttig, Appl. Phys. Lett. 84, 5240 (2004).

${ }^{24}$ P. Zalden, A. von Hoegen, P. Landreman, M. Wuttig, and A. M. Lindenberg, Chem. Mater. 27, 5641 (2015).

${ }^{25}$ F. Mallamace, C. Branca, C. Corsaro, N. Leone, J. Spooren, S.-H. Chen, and H. E. Stanley, Proc. Nat. Acad. Sci. 107, 22457 (2010).

${ }^{26}$ L. Battezzati and A. L. Greer, J. Mater. Res. 3, 570 (1988).

${ }^{27}$ S. Stølen, T. Grande, and H.-B. Johnsen, Phys. Chem. Chem. Phys. 4, 3396 (2002).

${ }^{28}$ B. Kalkan, S. Sen, J.-Y. Cho, Y.-C. Joo, and S. M. Clark, Appl. Phys. Lett. 101, 151906 (2012).

${ }^{29}$ M. Kalyva, J. Orava, A. Siokou, M. Pavlista, T. Wagner, and S. N. Yannopoulos, Adv. Funct. Mater. 23,2052 (2013).

${ }^{30}$ G. Eising, T. van Damme, and B. J. Kooi, Cryst. Growth Des. 14, 3392 (2014).

${ }^{31}$ C. Way, P. Wadhwa, and R. Busch, Acta Mater. 55, 2977 (2007). 
${ }^{32}$ H. L. Luo, Ph. D. Thesis, California Institute of Technology, 1964.

${ }^{33}$ G. C. Sosso, J. Behler, and M. Bernasconi, Phys. Status Solidi A 213, 329 (2016).

${ }^{34}$ S. Wei, P. Lucas, and C. A. Angell, J. Appl. Phys. 118, 034903 (2015).

${ }^{35}$ C. Zhou, L. Hu, Q. Sun, H. Zheng, C. Zhang, and Y. Yue, J. Chem. Phys. 142, 064508 (2015).

${ }^{36}$ J. Orava and A. L. Greer, J. Chem. Phys. 140, 214504 (2014).

${ }^{37}$ Q. Wang, L.-M. Wang, M. Z. Ma, S. Binder, T. Volkmann, D. M. Herlach, J. S. Wang, Q. G. Xue, Y. J. Tian, and R. P. Liu, Phys. Rev. B 83, 014202 (2011).

${ }^{38}$ S. Gruner and W. Hoyer, J. Alloy. Comp. 480, 629 (2009).

${ }^{39}$ R. Roscoe and W. Bainbridge, Proc. Phys. Soc. 72, 576 (1958).

${ }^{40}$ R. F. Brooks, A. T. Dinsdale, and P. N. Quested, Meas. Sci. Technol. 16, 354 (2005).

${ }^{41}$ T. Iida and R. I. L. Guthrie, The Physical Properties of Liquid Metals (Clarendon Press, Oxford, 1988).

${ }^{42}$ D. R. Lide, CRC Handbook of Chemistry and Physics, $89^{\text {th }}$ ed. (CRC Press, Boca Raton, 2008-2009). 